\title{
Role of Macrophages During early Achilles Tendon Healing
}

\author{
P. Blomgran ${ }^{1}$, M. Hammerman ${ }^{1}$, R. Blomgran², P. Aspenberg ${ }^{1,3}$ \\ ${ }^{1}$ Department of Clinical and Experimental Medicine, Faculty of Medicine and Health Sciences, Linkoping \\ University, Sweden \\ 2 Department of Biomedical and Clinical Sciences, Faculty of Medicine and Health Sciences, Linkoping University, \\ Sweden \\ ${ }^{3}$ Deceased
}

\author{
CORRESPONDING AUTHOR: \\ Parmis Blomgran \\ Department of Clinical \\ and Experimental Medicine \\ Faculty of Medicine \\ and Health Sciences \\ Linkoping University \\ 58185 Linkoping, Sweden \\ E-mail: parmis.blomgran@gmail.com \\ DOI: \\ 10.32098/mltj.01.2021.02
}

LEVEL OF EVIDENCE: 2

\begin{abstract}
SUMMARY
Background. Macrophages are a heterogeneous cell population that plays an important role in the initiation of the inflammatory response to trauma as well as its resolution during healing. However, their role during Achilles tendon healing is unclear. The aim of this study was to investigate if macrophage reduction by using clodronate liposome injection would influence the mechanical properties of the healing tendon.

Methods. The right Achilles tendon of 46 rats were transected and left to heal spontaneously (day 0 ). The reduction of macrophages during the inflammatory phase of tendon healing was studied by injecting clodronate liposomes day - 3, - 1 and 1 . To study the early remodeling phase, clodronate was injected day 3,5 and 7 . The controls received saline and the rats were evaluated by mechanical testing day 7 and 12, respectively.

Results. Clodronate injections during the inflammatory phase increased transverse area $(\mathrm{p}$ $=0.006)$ and stiffness $(\mathrm{p}=0.044)$ day 7 . In contrast, no significant effects were seen at day 12. Flow cytometry evaluation confirmed reduction of mature and polarized macrophages. Conclusions. Reduction of macrophages during the inflammatory phase of Achilles tendon healing influenced the mechanical properties, suggesting a regulatory role of macrophages during this phase.
\end{abstract}

\section{KEY WORDS}

Macrophage; inflammation; tendon bealing; immune cells; rat model.

\section{INTRODUCTION}

Tendon healing starts with an inflammatory phase, where the leukocytes $\left(\mathrm{CD} 45^{+}\right)$are recruited to the site of injury (1). Neutrophils are the first cells that arrive at the site of injury, followed by macrophages. Macrophages are heterogeneous cell population that, among other functions, can orchestrate the healing processes. They are present during the different phases of tendon healing and can become polarized to either M1 or M2 macrophages. Classically activated macrophages (M1) are pro-inflammatory and promote matrix degradation. Alternatively, activated macrophages (M2) are anti-inflammatory, stimulates cell proliferation and production of extracellular matrix, thereby stimulating repair of the injured tendon. However, M1 and M2 macrophages are a crude classification for what in reality is a spectrum of polarized macrophages. Macrophages are the dominant immune cells during the inflammatory phase, as almost half of all leukocytes are macrophages during the first 3 days after tendon injury (1). This suggests that macrophages are one of the most important players during inflammation and tendon healing.

Furthermore, in vivo studies show that non-steroidal anti-inflammatory drugs like parecoxib and indomethacin impair healing of tendon dramatically if given during the inflammatory phase but have a slight positive effect if given during the remodeling phase $(2,3)$. However, it seems that these effects on the mechanical properties of the healing tendon are orchestrated mainly via mechanisms not directly related to the amount of inflammatory cells, because there was no effect of parecoxib on the numbers of macrophages and their subtypes during the inflammatory and remodeling phases (4). Corticosteroids also influence Achilles tendon 
healing. Early dexamethasone treatment during the inflammatory phase had a negative effect on healing tendon. In contrast, its application during the early remodeling phase improved the tendon healing dramatically. Similar to NSAIDs, corticosteroids do not seem to change macrophage numbers, since dexamethasone treatment during the inflammatory and early remodeling phase did not have any effect on percentage of macrophages subpopulations compared to control. Still, there was a dramatic effect on mechanical properties (5). This raises the question how dampening and influencing the inflammation via macrophages with different treatments would affect the outcome in the healing tendon.

The primary aim of this study was to see if reduction of mature macrophages, using clodronate liposomes in an Achilles tendon healing model would influence the mechanical properties of the healing tissue. The secondary aim was to see which healing phase was most sensitive to clodronate liposomes, the inflammatory or the early remodeling phase.

To test this hypothesis, we used serial intraperitoneal injections of liposome-encapsulated dichloromethylene-bisphosphonate (clodronate liposome).

\section{MATERIALS AND METHODS}

\section{Study design}

46 female Sprague-Dawley rats (11-12 weeks old) were included in the study. The right Achilles tendon was transected in all rats and allowed to heal spontaneously without suture. Rats were randomized to clodronate liposome treatment or saline control. The day of surgery was regarded as day 0 .

To evaluate the mechanical properties of the healing tendon, rats received clodronate liposome or saline day - 3, - 1 and +1 and were euthanized day 7 , or received clodronate liposome or saline day 3, 5, 7 and were euthanized day 12 .

For flow cytometric analysis, animals received clodronate or saline day - 3, - 1 and +1 and were euthanized at day 7 .

The rats were housed 2 per cage with 12:12 light and given food and water ad libitum. All experiments were approved by the Regional Ethics Committee (15 15) and were in accordance with the MLTJ guidelines (6).

\section{Surgery}

Rats were anesthetized with isoflurane gas (Forene, Abbot Scandinavia, Solna, Sweden) and received antibiotics (25 $\mathrm{mg} / \mathrm{kg}$, Oxytetracycline, Engemycin; Intervet, Boxmeer, The Netherlands) preoperatively and analgesics (0.045 $\mathrm{mg} / \mathrm{kg}$, Buprenorphine, Temgesic; Schering-Plough, Brussels, Belgium) was given subcutaneously pre- and post-operatively. The skin on the right Achilles tendon was shaved and cleaned with chlorhexidine ethanol. Thereafter, a transverse skin incision was made lateral to the Achilles tendon. The plantaris tendon was removed and the Achilles tendon was cut transversely, and the tendon was left unsutured to heal spontaneously. The skin was sutured with two stitches.

\section{Clodronate liposome administration}

Rats were randomized to receive intraperitoneal injection of clodronate liposomes (Clodronate Liposomes, Amsterdam, Netherlands) or saline. $1.5 \mathrm{ml}$ clodronate or saline was injected every injection day, i.e., day - 3, - 1 and +1 or day 3,5 and 7 .

\section{Mechanical evaluation}

Rats were euthanized by $\mathrm{CO}_{2}$. The Achilles tendon with the calcaneal bone and gastrocnemius and soleus muscles was harvested. The muscle was scraped off and tendon fibers were fixed by sand paper in a metal clamp. The calcaneal bone was fixed in a custom-made clamp at $30^{\circ}$ dorsiflexion relative to the direction of traction. The mechanical testing machine (100R, DDL, Eden Praire, MN) pulled the mounted tendon at constant speed $(0.1 \mathrm{~mm} / \mathrm{s})$ until failure. Peak force at failure $(\mathrm{N})$, stiffness $(\mathrm{N} / \mathrm{mm})$, and energy uptake $(\mathrm{Nmm})$ were calculated by the testing machine. Sagittal and transverse diameter of the mid part of the callus was measured by a caliper. Transverse area, Young's modulus and peak stress were calculated assuming an elliptic cylindrical shape (5). All measurements were performed by a blinded investigator.

\section{Tissue harvest and retrieval of single cells}

To evaluate the efficacy of clodronate and overall effect on the immune signature during treatment, the spleen from same animals undergoing tendon mechanical testing at day 7 was used for flow phenotyping. The harvested spleen was minced in digestion buffer (RPMI 1640 with, 5\% heat inactivated fetal bovine serum, and $10 \mathrm{mM} \mathrm{HEPES}$ ) and passed through a $70 \mu \mathrm{m}$ cell strainer (Fisher scientific) to extract single cells. RBC was removed using ACK lysis buffer $(155 \mathrm{mM} \mathrm{NH} 3 \mathrm{Cl}, 10 \mathrm{mM} \mathrm{KHCO} 3$, and 88 $\mu \mathrm{M}$ EDTA) and cells were washed using digestion buffer. Trypan blue (Life technologies) was used to count live cells, and 500.000 cells per staining reaction were used for immune cell phenotyping as described below. 
Flow cytometric phenotyping of immune cells

Antibodies were CD45-PE-Cy7 (leukocyte), CD3-AF647 (T cell), CD4-PE (T helper cell), CD8-PerCP (cytotoxic T cell) from Biolegend and CD11b-AF700 (phagocyte), CD68BV510 (pan-macrophage) from AbD serotec and CCR7AF647 (M1 macrophage), CD206-FITC (M2a macrophage) from Bioss and CD163-PE (M2c macrophage) from LSBio. Single cell suspensions were first stained with $50 \mu \mathrm{l}$ antibody mixture for the surface markers CD45, CD11b, CD163, CD206, CCR7 (macrophage panel), or CD45, CD3, CD4, $\mathrm{CD} 8$ ( $\mathrm{T}$ cell panel) for 30 minutes in room temperature. Cells were then washed once and incubated with Cytofix/ Cytoperm for intracellular staining according to manufacturer's instructions (BD Biosciences) at $4{ }^{\circ} \mathrm{C}$ for $20 \mathrm{~min}$. Fixed and permeabilized samples were stained with intracellular marker CD68 diluted in Perm/Wash buffer for 30 minutes. Cells were then washed 3 times with Perm/ Wash buffer before analysis (1). Data were acquired using a Gallios flow cytometer (Beckman Coulter) equipped with a $405 \mathrm{~nm}, 488 \mathrm{~nm}, 633 \mathrm{~nm}$ laser and analyzed according to our previous experience using these antibodies for tendon tissue samples (4). Evaluation was performed using FlowJo v10 software (Treestar, Ashland, OR).

\section{Statistics}

The mechanical parameters were tested by student's t-test for testing the hypothesis of an effect of clodronate injections. Flow cytometric data were analyzed by students t-test to describe the response to clodronate injection in polarized macrophages.

\section{RESULTS}

\section{Mechanical evaluation}

We transected the right Achilles tendon on rats, and randomized them to either clodronate liposome or saline injections day - 3, - 1 and +1 and performed mechanical evaluation at day 7. Clodronate injections increased transverse area by $36 \%(\mathrm{p}=0.006)$, peak force by $24 \%(\mathrm{p}=0.083)$ and stiffness by $22 \%(\mathrm{p}=0.044)$ (figure 1 , table I).

Next, we transected the right Achilles tendon in another group of rats and this time we gave them clodronate liposome or saline days 3, 5, 7 and performed mechanical evaluation at day 12. With this treatment timing, clodronate increased transverse area by $16 \%$ and stiffness by $7 \%$, but these differences were not statistically significant (table I).

\section{Flow cytometric evaluation}

In order to see how clodronate injections affected the immune cell populations, we performed comprehensive flow cytometric analysis on spleen samples from rats treated with clodronate days $-3,-1,+1$ and euthanized day 7 . There was a statistically significant reduction in CCR7 (M1 macrophages; $\mathrm{p}<$ 0.001), CD163 (M2 macrophages; $\mathrm{p}=0.003$ ) and CD206 (M2 macrophages; $p=0.005)$ in the clodronate treated animals (figure 2). To determine the specificity of the treatment and if macrophage reduction by clodronate have a compensatory effect on $\mathrm{T}$ cells and their subtypes this was evaluated using the same specimens. There was no effect on frequency of $\mathrm{T}$ cells (CD3) or their subtypes, T helper cells (CD4) and cytotoxic $\mathrm{T}$ cells (CD8), by clodronate treatment (figure 3 ).

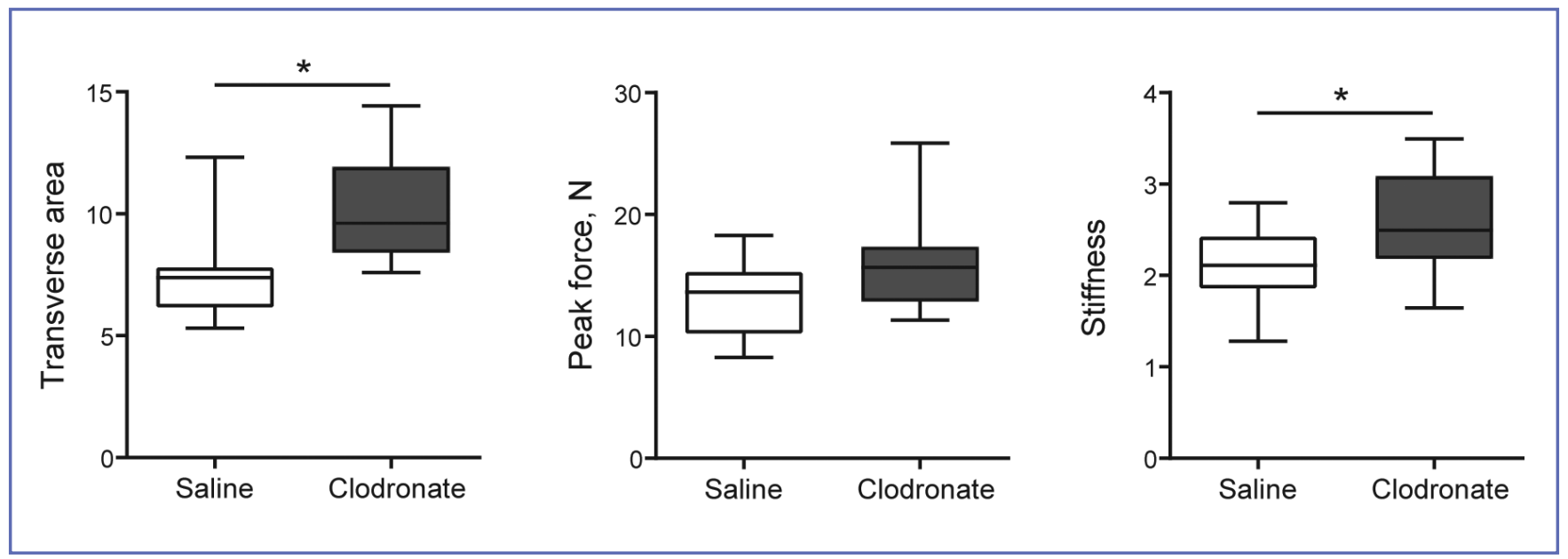

Figure 1. Reduction of macrophages influences mechanical properties of the healing Achilles tendon. Transverse area, peak force and stiffness was measured at day 7 after tendon transection. Animals received clodronate or saline days $-3,-1,+1 . \mathrm{N}$ $=10-12$ rats in each group. 
Table I. Mechanical properties of rat Achilles tendons at 7 and 12 days after tendon transection. Values are expressed as means (SD).

\begin{tabular}{llrrr}
\hline & & Saline (SD) & Clodronate (SD) & p-value \\
\hline Day 7 & Transverse area $\left(\mathrm{mm}^{2}\right)$ & $7.5(1.9)$ & $10.2(2.1)$ & 0.006 \\
& Peak force $(\mathrm{N})$ & $13.0(3.1)$ & $16.2(4.7)$ & 0.083 \\
& Stiffness (N/mm) & $2.1(0.4)$ & $2.6(0.6)$ & 0.044 \\
& Peak stress (MPa) & $1.8(0.3)$ & $1.6(0.4)$ & 0.309 \\
& Elastic Modulus (MPa) & $4.0(0.4)$ & $3.7(1.1)$ & 0.415 \\
& Energy (Nmm) & $28.1(11.1)$ & $38.1(13.8)$ & 0.080 \\
\hline Day 12 & Transverse area (mm²) & $10.4(2.3)$ & $12.1(2.8)$ & 0.160 \\
& Peak force (N) & $35.6(8.9)$ & $39.8(5.5)$ & 0.216 \\
& Stiffness (N/mm) & $6.0(1.3)$ & $6.4(0.9)$ & 0.378 \\
& Peak stress (MPa) & $3.5(0.97)$ & $3.4(0.9)$ & 0.839 \\
& Elastic Modulus (MPa) & $8.1(1.9)$ & $7.6(2.6)$ & 0.647 \\
& Energy (Nmm) & $67.2(19.4)$ & $77.7(15.9)$ & 0.204 \\
\hline
\end{tabular}

A
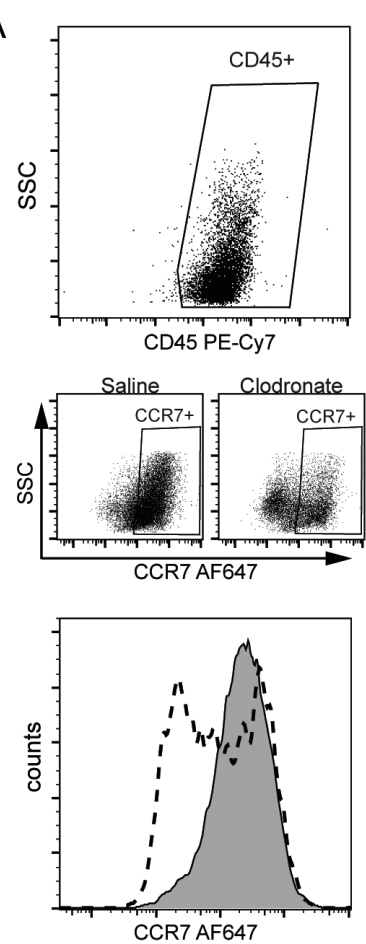

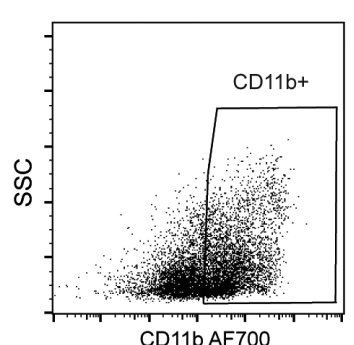

CD11b AF700
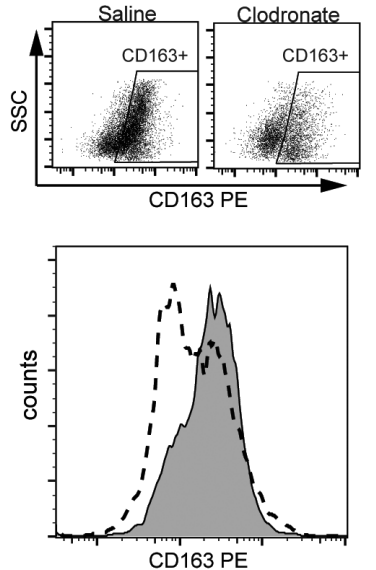

CD163 PE

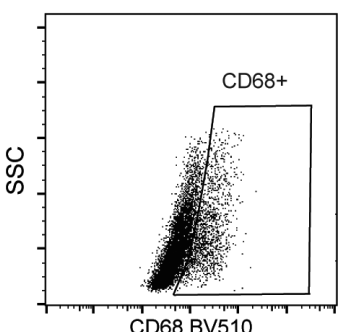

CD68 BV510
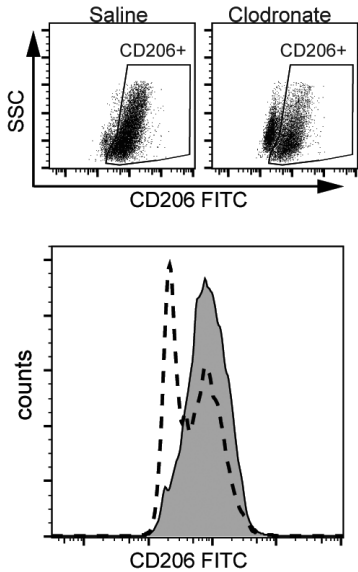

B

Saline Clodronate

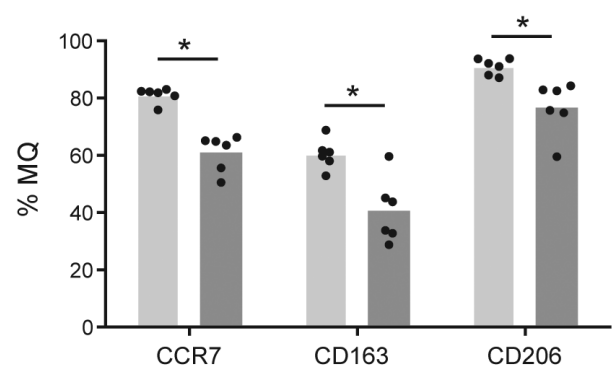

Figure 2. Decrease in polarized macrophages during early clodronate treatment. Flow cytometry was used to quantify the different macrophage subtypes in the spleen 7 days after tendon transection. Animals received clodronate or saline days - 3, 1, + 1. (A) Gating strategy for macrophages (CD45 ${ }^{+}$CD11 $b^{+}$ $\mathrm{CD}^{2} 8^{+}$cells), and representative dot plots for CCR7, CD163, CD206 positive macrophages, as well as histogram with overlay of saline (solid line, grey color) and clodronate (dashed line). (B) Bar graphs of CCR7, CD163, CD206 positive macrophages showing mean and each individual animal as a dot. $N=6$ in each group. 
A

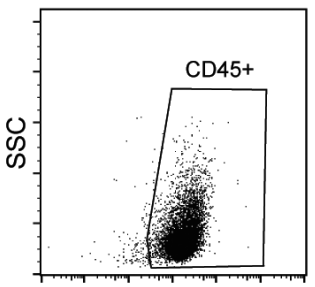

CD45 PE-Cy7

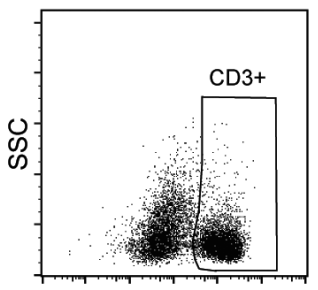

CD3 AF647

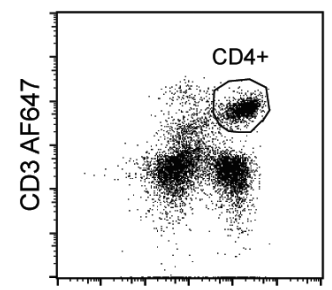

CD4 PE

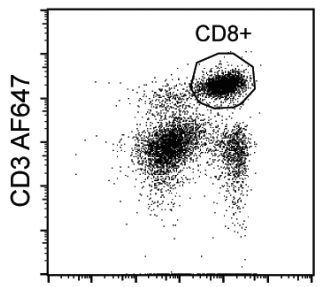

CD8 PerCP

B

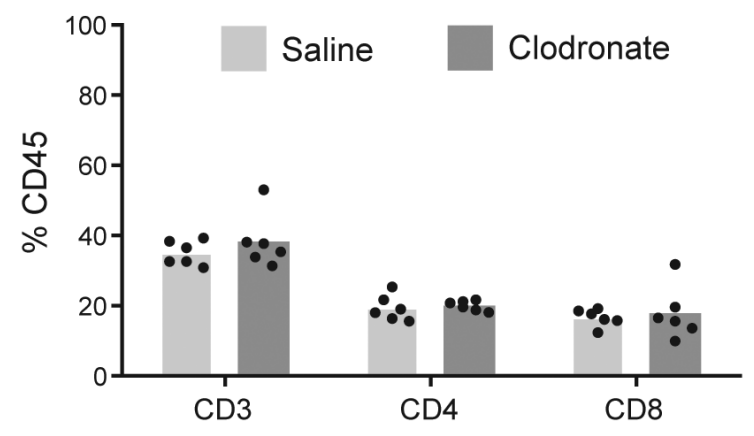

Figure 3. Unaffected T cells during early clodronate treatment. Flow cytometry was used to quantify $T$ cell subpopulations in the spleen 7 days after tendon transection. Animals received clodronate or saline days - 3, - 1, + 1. (A) Gating strategy for CD4 and CD8 positive T cells $\left(C D 45^{+} C D 3^{+}\right)$. (B) Bar graphs of total T cells and CD4 and CD8 $T$ cells showing mean and each individual animal as a dot. $N=6$ in each group.

\section{DISCUSSION}

Inflammation is an important phase of the healing process. Macrophages play a role both in the initiation and the resolution of inflammation, and they are considered the major immune cells involved in healing. Significant infiltration of macrophages into the tendon tissue has been shown in murine and human studies (1, 7-11). In this study, we investigated the role of macrophages during different phases of Achilles tendon healing by using clodronate liposomes.

Injections of clodronate liposomes days - 3, - 1, + 1 increased the mass of the healing tissue (transverse area) as well as stiffness and strength. Delayed clodronate liposomes injections day 3, 5 and 7 did not significantly influence the mechanical properties of tendon, although transverse area, stiffness and peak force seemed slightly increased. This suggests that macrophages play a role in tendon healing mainly during the early inflammatory phase.

Improvement of the healing tissue due to reduction of macrophages by clodronate has been shown previously in a tendon-to-bone repair study (12). They showed that the load to failure as well as stiffness was increased in rats treated with clodronate. Their histology evalua- tion showed that clodronate decreased accumulation of TGF- $\beta$ in the tendon-bone interface and this interface with fibrous tissue was narrower compared to controls. As TGF- $\beta$ promotes healing predominantly through scar formation (13), the decrease of TGF- $\beta$ due to macrophage reduction might lead to a wound healing process with reduced scar formation, which might improve the healing tissue. This suggests that macrophage reduction in our model could also had reduced TGF- $\beta$ levels which could explain the improvement of the mechanical properties, but this needs to be further investigated. In a mice model of Achilles tendon healing, clodronate liposome injection given during the inflammatory phase increased maximal stress and young's modulus measured at a delayed timepoint of 14 days after surgery (14). We have instead evaluated the healing outcome of clodronate shortly after treatment, both during the inflammatory phase and during early remodeling. Furthermore, a bone-healing model showed that clodronate liposome injections before the trauma had a significant effect on the mechanical properties of bone, while clodronate injections after the trauma did not have any impact (15). 
All these clodronate studies are different regarding the time points of clodronate injections which might explain the different results of the mechanical properties. Overall, the clodronate studies shows that macrophage reduction can have different effects on the healing process depending in which time point of the healing process the clodronate is given. The reason why we did not see an effect of macrophage reduction during the early remodeling phase in our study might be because clodronate only affects circulating monocytes/macrophages and the recruitment of these cells to the healing tendon at this time point might not be as high as during the inflammatory phase. The macrophages that were recruited during the inflammatory phase will stay in the healing tendon tissue also during the early remodeling phase and might not be affected by the clodronate injections given during this period. In summary, the clodronate studies done in tendon and bone healing shows that macrophages play a crucial role during the different phases of the healing process, and whether their reduction is good or bad is not yet clarified. Modulating the activity of macrophages rather than depleting them can also have an effect on tendon healing. Patellar tendon healing in $\mathrm{C} 3 \mathrm{H} / \mathrm{HeJ}$ mice with a genetic deficiency reducing macrophages ability to produce TNF and other inflammatory cytokines, showed that collagen fibril diameters were larger and more organized at 7 and 14 days post injury, suggesting that reduction of an inflammatory response improves quality of the healing tendon (16).

Clodronate injections in this study significantly reduced polarized macrophages during the inflammatory phase. The reduction was seen for both M1 (CCR7) and M2 macrophages (CD163 and CD206). Although, the reduction was only $20 \%$ in each subtype it had a big impact on the mechanical properties. This strengthen our conclusion that macrophages are important during early tendon healing as only

\section{REFERENCES}

1. Blomgran P, Blomgran R, Ernerudh J, Aspenberg P. A possible link between loading, inflammation and healing: Immune cell populations during tendon healing in the rat. Sci Rep 2016;6:29824.

2. Virchenko O, Skoglund B, Aspenberg P. Parecoxib impairs early tendon repair but improves later remodeling. Am J Sports Med 2004;32(7):1743-1747.

3. Forslund C, Bylander B, Aspenberg P. Indomethacin and celecoxib improve tendon healing in rats. Acta Orthop Scand 2003;74(4):465-469.

4. Blomgran P, Blomgran R, Ernerudh J, Aspenberg P. Cox-2 inhibition and the composition of inflammatory cell populations during early and mid-time tendon healing. MLTJ 2017;7(2):223-229. a $20 \%$ decrease of macrophages can change the mechanical properties of the healing tendon. Whether specifically targeting one subtype of macrophages (M1 or M2) would have a different outcome is still to be investigated.

We also confirmed that clodronate treatment did not alter the frequency of $\mathrm{T}$ cells, in order to rule out presence of a compensatory mechanisms acting during macrophage reduction. Clodronate had no effect on $\mathrm{T}$ cells in general (CD3) or their subtypes, Thelper cells (CD4) and cytotoxic $\mathrm{T}$ cells (CD8). The same effect has previously been shown in a ligament-healing model were clodronate did not affect total T cells (CD3) (17). In our tendon healing model, we have recently reported that CD8 $\mathrm{T}$ cell depletion did not influence mechanical properties of healing tendons at day 7 (anti-CD8 single injection given day - 1) and at day 12 (antiCD8 single injection given day 5) (18). This further emphasizes involvement of macrophages over that of $\mathrm{T}$ cells during early tendon healing.

\section{CONCLUSIONS}

In conclusion, polarized macrophage reduction by using clodronate liposome injections during the inflammatory phase of tendon healing increased the mass, the stiffness and probably the strength of the healing tissue.

\section{ACKNOWLEDGMENTS}

The study was funded by the Swedish Research Council. Per Aspenberg passed away before the submission of the final version of this manuscript.

\section{CONFLICT OF INTERESTS}

The authors declare that they have no conflict of interests.
5. Blomgran P, Hammerman M, Aspenberg P. Systemic corticosteroids improve tendon healing when given after the early inflammatory phase. Sci Rep 2017;7(1):12468.

6. Padulo J, Oliva F, Frizziero A, Maffulli N. Muscles, Ligaments and Tendons Journal - Basic principles and recommendations in clinical and field Science Research: 2018 Update. MLTJ 2018;8(3):305-307.

7. Klatte-Schulz F, Minkwitz S, Schmock A, et al. Different Achilles Tendon Pathologies Show Distinct Histological and Molecular Characteristics. Int J Mol Sci 2018;19(2):404.

8. Bergqvist F, Carr AJ, Wheway K, et al. Divergent roles of prostacyclin and PGE2 in human tendinopathy. Arthritis Res Ther 2019;21(1):74. 
9. Marsolais D, Cote CH, Frenette J. Neutrophils and macrophages accumulate sequentially following Achilles tendon injury. J Orthop Res 2001;19(6):1203-1209.

10. Xu HT, Lee CW, Li MY, Wang YF, Yung PS, Lee OK. The shift in macrophages polarisation after tendon injury: A systematic review. J Orthop Translat 2020;21:24-34.

11. Chisari E, Rehak L, Khan WS, Maffulli N. The role of the immune system in tendon healing: a systematic review. Br Med Bull 2020;133(1):49-64.

12. Hays PL, Kawamura S, Deng XH, et al. The role of macrophages in early healing of a tendon graft in a bone tunnel. $J$ Bone Joint Surg Am 2008;90(3):565-579.

13. Barrientos S, Stojadinovic O, Golinko MS, Brem H, Tomic-Canic M. Growth factors and cytokines in wound healing. Wound Repair Regen 2008;16(5):585-601.

14. de la Durantaye M, Piette AB, van Rooijen N, Frenette J. Macrophage depletion reduces cell proliferation and extra- cellular matrix accumulation but increases the ultimate tensile strength of injured Achilles tendons. J Orthop Res 2014;32(2):279-285.

15. Sandberg OH, Tatting L, Bernhardsson ME, Aspenberg P. Temporal role of macrophages in cancellous bone healing. Bone 2017;101:129-133.

16. Alaseirlis DA, Li Y, Cilli F, Fu FH, Wang JH. Decreasing inflammatory response of injured patellar tendons results in increased collagen fibril diameters. Connect Tissue Res 2005;46(1):12-17.

17. Chamberlain CS, Leiferman EM, Frisch KE, et al. The influence of macrophage depletion on ligament healing. Connect Tissue Res 2011;52(3):203-211

18. Bernhardsson M, Dietrich-Zagonel F, Tatting L, Eliasson P, Aspenberg P. Depletion of cytotoxic (CD8+) T cells impairs implant fixation in rat cancellous bone. J Orthop Res 2019;37(4):805-811. 